\title{
Recent advances of chitosan nanoparticles as drug carriers
}

This article was published in the following Dove Press journal:

International Journal of Nanomedicine

8 April 201I

Number of times this article has been viewed

\section{Jun Jie Wang' \\ Zhao Wu Zeng ${ }^{1,2}$ \\ Ren Zhong Xiao' \\ Tian Xie' \\ Guang Lin Zhou' \\ Xiao Ri Zhan' \\ Shu Ling Wang'}

'Research Center for Biomedicine and Health, Hangzhou Normal University, Hangzhou, Zhejiang, People's Republic of China; ${ }^{2}$ College of Pharmaceutical Sciences, Zhejiang Chinese Medical University, Hangzhou, Zhejiang, People's Republic of China
Correspondence: Zhao Wu Zeng Research Center for Biomedicine and Health, Hangzhou Normal University, 222 Wenyi Road, Hangzhou, Zhejiang 3100 I2, People's Republic of China $\mathrm{Tel}+86$ 57| $2886228 \mathrm{I}$

Fax +86 57I 28865630

Email artgreenking@I26.com
Abstract: Chitosan nanoparticles are good drug carriers because of their good biocompatibility and biodegradability, and can be readily modified. As a new drug delivery system, they have attracted increasing attention for their wide applications in, for example, loading protein drugs, gene drugs, and anticancer chemical drugs, and via various routes of administration including oral, nasal, intravenous, and ocular. This paper reviews published research on chitosan nanoparticles, including its preparation methods, characteristics, modification, in vivo metabolic processes, and applications.

Keywords: chitosan nanoparticle, drug delivery system, preparation, modification, application

\section{Introduction}

Many drugs have problems of poor stability, water insolubility, low selectivity, high toxicity, side effects, and so on. Good drug carriers play a significant role in resolving these problems. Chitosan nanoparticles are a drug carrier with wide development potential and have the advantage of slow/controlled drug release, which improves drug solubility and stability, enhances efficacy, and reduces toxicity. Because of their small size, they are capable of passing through biological barriers in vivo (such as the blood-brain barrier) and delivering drugs to the lesion site to enhance efficacy. ${ }^{1}$ Modified nanoparticles also have other properties such as improved drug targeting. Under the action of enzymes in vivo, biodegradable nanoparticles can produce water and carbon dioxide without adverse effects, and have thus become the focus of increasing research.

As a copolymer containing $\beta$-(1,4)-2-acetamido-D-glucose and $\beta$ - $(1,4)$-2-aminoD-glucose unit (Figure 1), chitosan is produced by removing an acetate moiety from chitin through hydration in concentrated alkali. Chitosan is soluble in most diluted acids. Under acidic conditions, chitosan can be dissolved in water after amino protonation to confer positive charges, gelation, and membrane-forming properties. The glycosidic bond of chitosan is hemiacetal, and thus is not stable in acid and will be hydrolyzed under acidic conditions, resulting in decreased viscosity and molecular weight of chitosan. Physical and chemical properties of chitosan depend mainly on its molecular weight and degree of deacetylation. ${ }^{2}$ As a natural product, chitosan is a renewable pharmaceutic adjuvant with good biocompatibility. Chitosan and its derivatives have strong potential for application as drug carriers. 


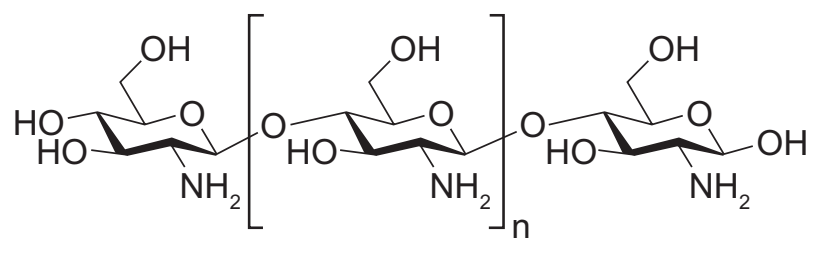

Figure I Structure of chitosan.

\section{Basic characteristics of chitosan Adhesivity of chitosan}

The amino and carboxyl groups in the chitosan molecule can be combined with glycoprotein in mucus to form a hydrogen bond, leading to an adhesive effect. As mucoprotein in mucus is positively charged, chitosan and mucus are attracted to each other to prolong the retention time of drugs and continuous drug release in vivo as well as improve drug bioavailability. ${ }^{2}$ Dudhani and Kosaraju, ${ }^{3}$ who prepared chitosan nanoparticles encapsulating catechin, found that encapsulated catechin had a release percentage of $60 \%$ under the action of enzyme. Release testing in vitro showed that $32 \%$ of catechin still existed after 24 hours. These results indicated that adhesivity of chitosan enhanced controlled release of catechin and improved its bioavailability. ${ }^{3}$ Adhesivity of chitosan is strengthened under neutral and acidic conditions. The greater the molecular weight and higher the degree of deacetylation of chitosan, the stronger will be its adhesivity and the amount of adhesion. Some chitosan groups (such as sulfydryl) can be easily modified to strengthen its adhesivity. ${ }^{2}$

\section{Biodegradability and safety of chitosan}

The biodegradability of chitosan is important for its use in drug delivery systems. Chitosan of suitable molecular weight can be cleared by the kidney in vivo, while that of excessive molecular weight can be degraded into fragments suitable for renal clearance. ${ }^{4}$

Chitosan is degraded mainly by chemical process and enzyme catalysis; the latter is the major in vivo process. ${ }^{4}$ The higher the degree of deacetylation, the greater the degradation rate. Degradation by enzyme catalysis also depends on the availability of chitosan's amino group. ${ }^{4}$ At present, it is generally believed that chitosan is a nontoxic polymer as well as safe drug delivery material. It has been certified as a wound dressings by the US Food and Drug Administration. ${ }^{5}$ Compared with toxic compounds such as sulfide $\left(\mathrm{LD}_{50}<20 \mu \mathrm{g} / \mathrm{mL}\right.$ against MCF7 and COS7 cells), chitosan has no obvious toxicity. However, with increasing charge density, toxicity of chitosan will strengthen accordingly. ${ }^{4}$ Intravenous injection of excessive chitosan may cause death due to blood coagulation. ${ }^{4}$

\section{Antitumor effect of chitosan}

Chitosan can act on tumor cells directly to interfere with cell metabolism, inhibit cell growth, or induce cell apoptosis. It also has an antitumor role through improving the body's immune function. ${ }^{6}$ Maeda and $\mathrm{Kimura}^{7}$ showed that low-molecular-weight chitosan and chito-oligosaccharide could inhibit tumor growth in S180-bearing mice. Torzsas et $\mathrm{al}^{8}$ found that a diet containing chitosan could reduce the generation of precancerous lesions in colon cancer induced by azomethane compounds. In vitro antitumor testing of chitosan nanoparticles indicated that inhibition rate of $500 \mathrm{mg} / \mathrm{L}$ chitosan nanoparticles was $27 \%$ on Hela cells of cervical cancer, $23 \%$ on liver SMMC-7721 cells, $29 \%$ on gastric cancer BGC-823 cells, and as high as $55 \%$ on breast cancer MCF-7 cells. ${ }^{9}$ These studies suggested that chitosan had antitumor effects in vitro and in vivo, leading to good prospects for their application as a supplementary antitumor drug and drug carrier. Studies have also indicated significant differences in antitumor activity of nanoparticles prepared by chitosan from different producers, and chitosan nanoparticles also had a selectivity for tumor cells. ${ }^{10}$

\section{Preparation techniques of chitosan nanoparticles lonic cross-linking}

As a widely used method for preparing chitosan nanoparticles, ionic cross-linking is generated by auto-aggregation between chitosan or its derivatives and macromolecules of opposite charge, or when ionic cross-linking agent exists. The most commonly used cross-linking agent is sodium tripolyphosphate. When it is added continuously to a water solution of chitosan with constant stirring under a moderate temperature, two components with opposite charges will combine to form nanoparticles. ${ }^{11}$ In the chitosan-Arabic gelatin nanoparticles of insulin prepared by Avadi et $\mathrm{al}^{12}$ minimum particle size and maximum combining rate with insulin were reached at a chitosan concentration of $10 \mathrm{mg} / \mathrm{mL}$, Arabic gelatin concentration of $5 \mathrm{mg} / \mathrm{mL}$, and insulin concentration of $5 \mathrm{mg} / \mathrm{mL} .{ }^{12}$ As ionic cross-linking can be performed at room temperature to avoid the use of organic solvent, and uniform nanoparticles with an adjustable size can be obtained easily, cross-linking has been widely used in encapsulation of protein and gene drugs. Using ionic cross-linking, chitosan nanoparticles were prepared by $\mathrm{Du}$ et $\mathrm{al}^{13}$ and then loaded with $\mathrm{Ag}^{+}, \mathrm{Cu}^{2+}, \mathrm{Zn}^{2+}, \mathrm{Mn}^{2+}$, and $\mathrm{Fe}^{2+}$. The zeta potential of chitosan nanoparticles is intensified significantly by positive charge of ions thus improving the stability of nanoparticles and greatly enhancing the antibacterial potential of nanoparticles. ${ }^{13}$ 


\section{Covalent cross-linking}

Chitosan and its derivatives can be prepared as nanoparticle drug carriers by using covalent cross-linking, ${ }^{14}$ which involves mainly the formation of covalent bonds between the chitosan chain and a functional cross-linking agent. This method was first used to prepare chitosan nanoparticles by encapsulating 5-fluorouracil by cross-linking glutaraldehyde with amino groups in the molecular chain of chitosan. ${ }^{15}$ Commonly applied agents also include polyethylene glycol (PEG) dicarboxylic acid, glutaraldehyde, or monofunctional agents such as epichlorohydrin. ${ }^{16,17} \mathrm{Fe}_{3} \mathrm{O}_{4}$-chitosan nanoparticles were prepared by Qu et a $1^{18}$ by covalent crosslinking (Figure 2). Oleic acid-coated $\mathrm{Fe}_{3} \mathrm{O}_{4}$ nanoparticles are absorbed by chitosan and cross-linked with glutaraldehyde, resulting in $\mathrm{Fe}_{3} \mathrm{O}_{4}$-chitosan nanoparticles of average size of $10.5 \mathrm{~nm}$ and a narrow size distribution. These nanoparticles have been shown to have a highly saturated magnetization effect, superparamagnetic properties, and a sufficiently high temperature to induce hyperthermia. ${ }^{18}$

\section{Precipitation}

There are two kinds of approaches for nanoparticle precipitation. One is desolvation, in which flocculant (commonly sodium sulfate) is added to a water solution of chitosan and solubility of chitosan is decreased by the combination of water and sodium sulfate, leading to the precipitation of nanoparticles due to hydrogen bonding between molecules. This method was first applied by Berthold et a $\mathrm{l}^{19}$ to prepare chitosan microspheres. Technical improvements then enabled Tian and Groves ${ }^{20}$ to prepare 600 - to 800 -nm chitosan nanoparticles. The other type is based on diffusion of emulsified solvent. Under the action of emulsified solvent, the water phase containing chitosan is dispersed in the organic phase
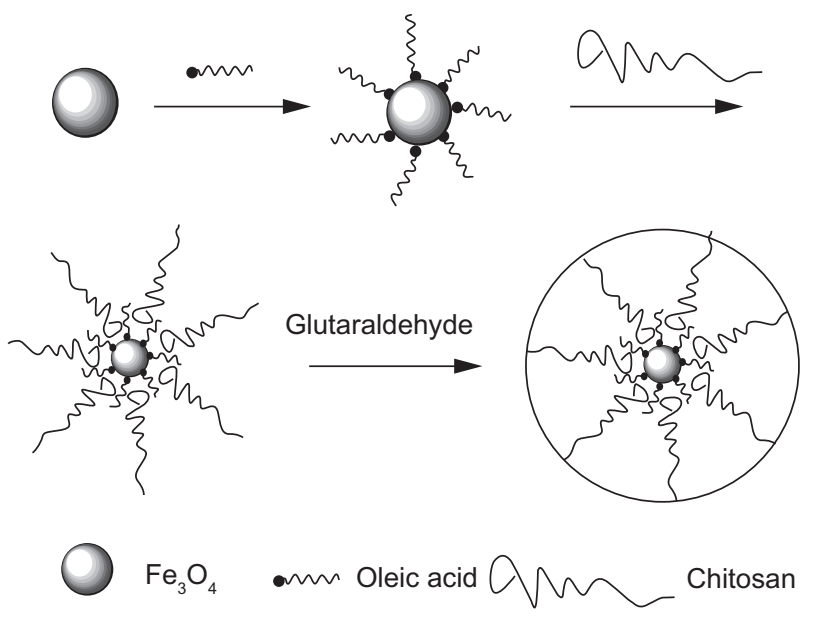

Figure 2 A schematic representation of $\mathrm{Fe}_{3} \mathrm{O}_{4}$-chitosan nanoparticles. ${ }^{18}$ encapsulating the drug, where turbulence appears between the interfaces of the two phases and chitosan is precipitated, resulting in the generation of nanoparticles..$^{21}$ In this method, organic solvent is used and the large nanoparticles obtained restricting their application.

\section{Polymerization}

Radical polymerization was initially developed by Chauvierre et al. ${ }^{22-24}$ It was later used to prepare chitosan and thiolated chitosan-poly (isobutyl cyanoacrylate) core-shell nanoparticles by Bravo-Osuna et al. ${ }^{25}$ Diluted nitric acid solution of chitosan was stirred continuously together with a mixed solution of ceric ammonium nitrate, nitric acid, and isobutyl cyanoacrylate at $40^{\circ} \mathrm{C}$ for 40 minutes in an argon environment. After cooling to room temperature, sodium hydroxide was added to adjust $\mathrm{pH}$ to 4.5 and a nanoparticle suspension was then obtained. ${ }^{25}$ Radical polymerization, by combining different monomers, can regulate both nanoparticle shell and core properties. de Moura et al ${ }^{26}$ prepared chitosan-methyl acrylic acid (CS-PMMA) nanoparticles by polymerization through ionic interaction between $\mathrm{COO}^{-}$produced by carboxyl dissociation from methyl acrylic acid and the amino group of chitosan. It was shown in a transmission electron microscope micrograph that these nanoparticles were uniformly spherical and quite consistent in size distribution.

\section{Self-assembly}

Amphiphilic compounds dispersed in water can form nanoparticles with a core-shell structure by self-assembly. With a hydrophobic core and a hydrophilic shell, amphiphilic nanoparticles can be used as carriers for hydrophobic and hydrophilic drugs simultaneously. The hydrophilic shell greatly reduces macrophage phagocytosis. Therefore, amphiphilic nanoparticles have attracted increasing attention. Kim et al ${ }^{27}$ used hydrophobic cholanic acid to modify glycol chitosan and then form nanoparticles by self-assembly in water solution. The antitumor drug cisplatin was encapsulated into the hydrophobic core with a drug loading of $80 \% .{ }^{28}$ The amphiphilic derivatives of N-octyl-O, N-carboxymethyl chitosan (OCC) prepared by Huo et a ${ }^{28}$ enhanced the solubility of the insoluble antitumor drug paclitaxel. Solubility of paclitaxel was improved nearly 500 -fold with a drug loading of $34.6 \%$ and encapsulation rate of $89.9 \%$, indicating that OCC is a potentially excellent solubilization carrier for insoluble drugs.

\section{Chitosan-drug complexes}

Because of the cationic properties of chitosan, ion complexes can be formed by electrostatic interaction between 
chitosan and anionic drugs. Cheng et $\mathrm{al}^{29}$ prepared chitosan nanoparticles linked with antisense oligonucleotide (asON). By the mixing method, the nanoparticles could be made easily under the optimal condition of 2:1 M proportion of chitosan phosphate and asON. The size of the nanoparticles was $102.6 \pm 12.0 \mathrm{~nm}$, their zeta potential was $1.45 \pm 1.75$, and the encapsulated ratio of chitosan cross-linked as $\mathrm{ON}$ was $87.6 \pm 3.5 \%$. Kim et $\mathrm{al}^{30}$ encapsulated retinol into chitosan nanoparticles through the electrostatic interaction between the amine group of chitosan and the hydroxyl group of retinol. Encapsulation into chitosan nanoparticles was shown to increase solubility of retinol by more than 1600-fold.

\section{Spray-drying}

Spray-drying method can be used as a one-step preparation of nanoparticle powder. Grenha et $\mathrm{al}^{31}$ prepared mannitol microspheres containing chisosan nanoparticles-loaded protein by this method. Huang et $\mathrm{al}^{32}$ prepared chitosaniron oxide nanoparticles with various chitosan:iron oxide ratios by spray-drying. Atomic absorption spectrometry results implied that chitosan had strong chelation with iron. Meanwhile, $\mathrm{Fe}_{3} \mathrm{O}_{4}$ was crystallized and distributed in the chitosan matrix. These chitosan-iron oxide nanoparticles were stable in water with strong superparamagnetism.

\section{Characteristics of chitosan nanoparticles General characteristics}

Nanoparticles are solid colloid particles of 1 to $1000 \mathrm{~nm}$. Compared with micron-grade particles, nanoparticles have strong mobility because of their small size and can enter cells easily to accumulate at the lesion site. Therefore, cell uptake rate is high. ${ }^{33}$

\section{Characteristics of sustained/controlled release}

Drugs carried by chitosan nanoparticles can be released through degradation and corrosion of chitosan, leading to a clear sustained-release effect. Because of varied degradation rate and time of chitosan of different molecular weight and degree of deacetylation degree, different types of nanoparticles can be used to regulate drug-release rate. Meanwhile, chitosan can also be modified to achieve the sustained/ controlled release. Chitosan-alumino-silicate nanoparticles prepared by Yuan et $\mathrm{al}^{34}$ had significant sustained/controlledrelease effects. $\mathrm{pH}$ of the environment and chitosan:aluminosilicate ratio also influence drug release.

\section{Targeting of chitosan nanoparticles}

Positive charges of chitosan have selective adsorption and neutralizing effects on the tumor cell surface. As a drug carrier, it has a targeting function to liver, spleen, lung, and colon. ${ }^{35}$ Ethylene chitosan nanoparticles had a prolonged circulating time in the blood with a high tumor-cell selectivity. ${ }^{36}$ Moreover, this kind of effect was more obvious in ethylene chitosan nanoparticles of large molecular weight. Doxorubicin-chitosan polymeric micelles had excellent drug-loading properties, were suitable for targeting the liver and spleen, and significantly reduced drug toxicity to the heart and kidney. ${ }^{37}$

\section{Modification of chitosan nanoparticles}

In order to improve targeting and bioavailability of chitosan nanoparticles, an increasing number of studies are focusing on modification of chitosan. Modified chitosan nanoparticles are characterized by $\mathrm{pH}$ sensitivity, thermosensitivity, and targeting accuracy.

\section{Modification of $\mathrm{pH}$ sensitivity}

A $\mathrm{pH}$-sensitive nanocarrier is a drug delivery system that increases drug release by changing carrier properties under a certain acid-base environment in vivo, and targets the lesion tissue. Poly(propyl acrylic acid) (PPAA) is a polymer that is highly sensitive to $\mathrm{pH}$. At a $\mathrm{pH}$ lower than 6.0, its high membrane fragmentation ability was shown to cause rupture of endosomal membrane and release vesicular materials into cytochylema. ${ }^{38}$ Therefore, Kiang et $\mathrm{al}^{39}$ added PPAA to chitosan-DNA complex to improve gene transfection efficiency. The results showed that adding PPAA to chitosanDNA complexes enhanced gene expression in both HEK293 and HeLa cells compared with chitosan nanoparticles alone. Camptothecine-loaded $\mathrm{N}$-isopropylacrylamide-chitosan nanoparticles were sensitive to $\mathrm{pH}$ : the nanoparticles were most sensitivite to tumor $\mathrm{pH}$ when the charge ratio of $\mathrm{N}$-isopropylacrylamide:chitosan was $4: 1{ }^{40}$ At a $\mathrm{pH}$ of 6.8 , cumulative release rate of camptothecine was highest and cell toxicity was significantly stronger. Cell toxicity was minimum at a $\mathrm{pH}$ of 7.4. Thus, $\mathrm{N}$-isopropylacrylamide-chitosan nanoparticles have a good potential for use as a targeted anticancer drug carrier. ${ }^{40}$ Chitosan nanoparticles prepared with sodium tripolyphosphate and glycidoxypropyltrimethoxysilane cross-linking were $\mathrm{pH}$ sensitive. ${ }^{41}$ With antihuman IgG antibody as a model protein drug, the release of antibody was increased from $5.6 \%$ to $50 \%$ when solution $\mathrm{pH}$ was adjusted from 7.4 to 6.0. Therefore, chitosan nanoparticles 
prepared by two-step cross-linking are a potential drug carrier sensitive to $\mathrm{pH} .{ }^{41}$

\section{Modification of thermosensitivity}

Drug release is regulated by structural change of thermosensitive drug carriers at different temperatures. Poly(N-isopropylacrylamide) is a well-known thermosensitive polymer widely used in drug carriers. ${ }^{42,43}$ Chitosanpolyvinylcaprolactan graft copolymer nanoparticles were sensitive to temperature, with a critical solution temperature at $38^{\circ} \mathrm{C} .{ }^{44}$ With 5-fluorouracil as a model drug, drug release mainly occurred above $38^{\circ} \mathrm{C}$ with high toxicity to tumor cells but low toxicity to normal cells.

\section{Modification of targeting}

Active targeting can be obtained in chitosan nanoparticles through chemical modification, so as to make the drug identify the target accurately. With resveratrol as a model drug, $\mathrm{Yao}^{45}$ prepared chitosan nanoparticles using ligands of both avidin and biotin to modify the nanoparticles. The resulting delivery system passively targeted the liver and positively targeted hepatoma cells. Two kinds of targeting mechanisms were thus combined in the new drug delivery system to achieve targeting to specific cells in specific tissues, further improving therapeutic effects and reducing toxic and side effects. ${ }^{45}$ Chitosan nanoparticles modified by glycyrrhizic acid, strengthened the active liver-targeting delivery of drug-loaded carriers through the mediation of glycyrrhizic acid because there were binding sites of glycyrrhizic acid on the surface of liver parenchyma. ${ }^{46} \mathrm{Kim}$ et $\mathrm{al}^{27}$ used hydrophobic cholanic acid to modify glycol chitosan and prepare nanoparticles through self-assembly. The antitumor drug cisplatin could be encapsulated easily in a hydrophobic core of nanoparticles. It was proven that due to prolonged circulating time in vivo and strengthened cell permeability and drug effect, drug-loaded nanoparticles were concentrated in tumor tissues of mice successfully with better antitumor effect and lower toxicity. ${ }^{27}$ Min et $\mathrm{al}^{47}$ used chemical binding to bind hydrophobic $5 \beta$-cholanic acid with the skeleton of hydrophilic glycol chitosan (HGC) to prepare nanoparticles. Using a dialysis method, camptothecine could be encapsulated into the nanoparticles easily, with drug-loading of more than $80 \%$. Camptothecine (CPT) was effectively protected from hydrolyzation by the hydrophobic core of HGC nanoparticles under a physiological environment, producing CPT-HGC nanoparticles with a significant antitumor effect and high targeting to MDA-MB231 human breast cancer cells. ${ }^{47}$ Hydrophobic glycol chitosan nanoparticles were shown to be excellent carriers for insoluble antitumor drugs.

\section{In vivo metabolic process of chitosan nanoparticles}

Nanoparticles are recognized as foreign matter in vivo and are absorbed by antibodies generated in the human body. Plasma protein, lipoprotein, immune protein, and complement $\mathrm{C}$ protein in plasma are also adsorbed on nanoparticles, accelerating the reorganization of the reticuloendothelial system. Nanoparticles are engulfed by macrophage and cleared from the body's circulation. ${ }^{48}$ The bridge between nanoparticles and macrophage is formed because of plasma protein adsorbed on the nanoparticle surface. The ability of nanoparticles to adsorb plasma protein is determined by surface charge of nanoparticles, thereby influencing the transfer intensity of nanoparticles by macrophage. ${ }^{49}$ Nanoparticles with polarity and high surface potential as well as amphipathic or hydrophilic nanoparticles are engulfed less and have a longer circulating time in vivo. Nam et al ${ }^{50}$ found that glycol-chitosan nanoparticles after hydrophobic modification are more distributed in all cells compared with unmodified nanoparticles.

Drug-loaded chitosan nanoparticles are decomposed into free chitosan and drug in vivo. Drugs enter cell and targeted tissues to generate therapeutic effects. Chitosan is mainly degraded under catalysis of lysozyme and bacterial enzyme in the colon. The chitosan absorbed into blood is cleared by the kidney and the rest is discharged through excrement. Degree of deacetylation as well as molecular weight also influence degradation rate and degree of chitosan in vivo. ${ }^{51,52}$

\section{Application of chitosan nanoparticles}

Studies have shown that chitosan nanoparticles can carry many drugs including gene drugs, protein drugs, anticancer chemical drugs, and antibiotics, and via various routes of administration including oral, nasal, intravenous, and ocular.

\section{Carrier for various drugs} Carrier of gene drugs

As a gene carrier, conventional virus has the disadvantages of low transfection rate and cell toxicity, ${ }^{53}$ and even causes serious immune response. As a nonvirus carrier, chitosan has excellent biocompatibility and biodegradation, which has led to increasing application of chitosan nanoparticles in gene drug delivery. ${ }^{54,55}$ Gene silencing mediated by double-stranded small interfering RNA (siRNA) has been 
widely investigated as a potential therapeutic approach for diseases caused by genetic defects. ${ }^{54}$ However, its application is restricted by rapid degradation and poor cell absorption. Drug loading of chitosan nanoparticles prepared by ionic gelation by Katas and Alpar ${ }^{54}$ reached $100 \%$, protecting well siRNA from nuclease degradation. With natural positive ion chitosan as a carrier material and using electrostatic interaction of polyelectrolyte, siRNA of silencing green fluorescent protein was compounded directly by $\mathrm{Liu}^{55}$ to prepare stable siRNA nanoparticles with a complex rate of $83 \%$ to $94 \%$. It was also found that more stable nanoparticles with positive surface charges could be generated by siRNA and chitosan with high molecular weight and degree of deacetylation. The product was not only easily adsorbed onto the cell surface to increase chance of cellular endocytosis, but also could protect siRNA activity effectively during the transfection in cells to improve gene silencing efficiency. However, gene transfection efficiency of chitosan nanoparticles is low generally and also influenced by molecular weight, degree of deacetylation, the chitosan:DNA ratio, environmental $\mathrm{pH}$, and nanoparticle preparation method. ${ }^{56}$ Improving transfection efficiency is a challenge for using chitosan nanoparticles as a gene carrier. Transfection efficiency of chitosan with different degrees of deacetylation and molecular weights was studied by Lavertu et al, ${ }^{57}$ who found that maximum transgene expression occurred when the ratio of the degree of deacetylation (DDA) to the molecular weight (MW) moves from high DDA/low MW to low DDA/high MW. Moreover, several chitosan-pDNA (plasmid DNA) complex formulations achieved levels of transgene expression approaching those of the positive controls (Lipofectamine ${ }^{\mathrm{TM}}$, Life Technologies, Carlsbad, CA; and FuGENE ${ }^{\circledR}$ 6, Roche Diagnostics, Basel, Switzerland), while two optimal conditions (92-10-5:1 and 80-10-10:1 [DDA-MW-N:P ratio] both at $\mathrm{pH}$ 6.5) were particularly effective, showing equivalent transfection efficiency compared with the best positive control. ${ }^{57}$ This provided a good example for the application of chitosan nanoparticles in gene transfection. Moreover, chitosan could also be modified by folic acid to improve transfection efficiency. Folic acid could be easily absorbed by cells, promoting the targeting and internalization of drug. Mansouri et $\mathrm{al}^{58}$ used folic acid to modify chitosan for improving gene transfection efficiency. They studied systematically the characteristics of folic acid for gene treatment, finding that folic acid-modified chitosan nanoparticles had low cell toxicity and could condense DNA effectively with ideal size and zeta potential. The results showed that folic acid-modified chitosan nanoparticles were a nonvirus gene carrier with a good application potential.

\section{Carrier of protein drugs}

Protein drugs can be degraded easily by enzymes in vivo and have poor permeability and stability as well as a short half-life. However, chitosan can protect protein well and promote the contact between drug and biomembrane, thereby improving bioavailability. ${ }^{11}$ Gan and Wang ${ }^{59}$ showed that changing the size and surface charge of chitosan-bovine serum albumin nanoparticles could regulate the encapsulation efficiency and release kinetics of bovine serum albumin, but it was difficult to control the burst release of protein of high molecular weight. Zhang et $\mathrm{al}^{60}$ used insulin and cationic $\beta$-cyclodextrin to form a complex encapsulated into alginate-chitosan nanoparticles. Binding rate and drug-loading amount were $87 \%$ and $9.5 \%$, respectively, and cumulative release of insulin in simulated intestinal fluid reached $40 \%$. Insulin was protected well in the nanoparticle core, avoiding the degradation in simulated gastric fluid, as well as the structure of insulin during release. Glycol chitosan nanoparticles modified by $5 \beta$-cholanic acid (HGC) and RGD (Arg-Gly-Asp) polypeptide were easily encapsulated into nanoparticles with a drug-loading amount greater than $85 \% .{ }^{61}$ RGD-HGC nanoparticles showed a one-week sustainedrelease effect. RGD-HGC displayed antiangiogenic efficacy by inhibiting human umbilical venous cord endothelial cell adhesion to a $\beta$ ig-h3 protein-coated surface, markedly suppressing bFGF-induced angiogenesis as well as decreasing hemoglobin content in Matrigel plugs in vivo. Therefore, RGD-HGC nanoparticles can inhibit tumor cell growth and reduce microvessel density significantly. ${ }^{61}$

\section{Carrier of anticancer chemical drugs}

Chitosan itself has a certain antitumor activity and its positive charge can neutralize the negative charge on the tumor cell surface, resulting in selective absorption. Thus, chitosan nanoparticles can increase drug concentration in the tumor site and improve therapeutic effects. Doxorubicin/methoxy PEG grafted carboxymethyl chitosan nanoparticles with higher cell toxicity could enter cell and inhibit tumor-cell proliferation effectively. ${ }^{62}$ Paclitaxel chitosan nanoparticles had a high encapsulation rate of $94.0 \% \pm 16.73 \%$ with sustained-release effect. ${ }^{63}$ Cell toxicity testing showed that paclitaxel-chitosan nanoparticles had a higher toxicity than that of paclitaxel alone, and with a higher cell uptake rate. Using an evaporation method of composite microemulsion 
solvent, Trickler et al ${ }^{64}$ combined glyceryl monooleate (GMO) and chitosan to prepare nanoparticles with a hydrophobic core and a hydrophilic shell. When encapsulated in the nanoparticle, paclitaxel had clear sustained-release characteristics; cell uptake increased four-fold and median lethal dose $\left(\mathrm{IC}_{50}\right)$ of paclitaxel decreased 1000-fold, leading to a maximum reduction of the side effects of paclitaxel. Kim et $\mathrm{al}^{65}$ linked $5 \beta$-cholanic acid to the main chain of glycol chitosan for the preparation of amphiphilic HGC nanoparticles. Encapsulated into nanoparticles by the dialysis method, paclitaxel had a drug-loading amount greater than $80 \%$. With significant sustained-release effect, paclitaxelHGC nanoparticles had low toxicity to B16F10 melanoma cells but a clear anticancer cell effect.

\section{Carrier of other drugs}

Chitosan nanoparticles also can load other drugs including antivirus drugs, antiallergic drugs, and hormone drug. Hao and Deng ${ }^{66}$ prepared acyclovir-loaded chitosan nanoparticles with a drug loading of $17.8 \%$ and an encapsulation rate of $87.5 \%$ by an ionic cross-linking method. Li and $\operatorname{Luan}^{67}$ prepared tranilast-loaded chitosan nanoparticles for allergic diseases with a particle size of $285.5 \mathrm{~nm}$ and an encapsulation rate of $82.4 \%$.

\section{Routes of administration}

Routes of administration of chitosan nanoparticles have been developed. Oral nanoparticles can protect drugs from degradation in the gastrointestinal tract and improve drug absorption. ${ }^{68} \mathrm{Yin}$ et a ${ }^{69}$ developed a promising vehicle for oral delivery. Trimethyl chitosan-cysteine conjugate (TMC-Cys) was synthesized in an attempt to combine the mucoadhesion- and the permeation-enhancing effects of TMC and thiolated polymers related to different mechanisms for oral absorption. The TMC-Cys nanoparticles, obtained via selfassembly, possessed spherical morphology, uniform size, positive zeta potentials, and high insulin encapsulation efficiency. Mucoadhesion and permeation enhancing effects of TMC-Cys nanoparticles were significantly higher than those of TMC nanoparticles. Biocompatibility assessment revealed lack of toxicity of TMC-Cys nanoparticles ${ }^{69}$ Because of poor stability and intestinal absorption of catechins, Dube et $\mathrm{al}^{70}$ encapsulated (+)-catechin (C) and (-)-epigallocatechin gallate (EGCg) in chitosan nanoparticles. The encapsulation significantly enhanced intestinal absorption and the cumulative amounts transported after encapsulation were significantly higher. ${ }^{70}$ An insulin-loaded, $\mathrm{pH}$-sensitive chitosan nanopar- ticle was formulated by ionic cross-linking with hydroxypropyl methylcellulose phthalate (HPMCP) as a $\mathrm{pH}$-sensitive polymer. ${ }^{71}$ In vitro results revealed a superior acid stability of CS-HPMCP nanoparticles with a significant control over insulin release and degradation in simulated acidic conditions with or without pepsin. Moreover, fluorescently labeled CS-HPMCP nanoparticles showed a 2- to 4-fold improvement in the intestinal mucoadhesion and penetration compared with CS-TPP nanoparticles. ${ }^{71}$ Amidi et al ${ }^{72}$ investigated the potential of N-trimethyl chitosan (TMC) nanoparticles as a carrier system for the nasal delivery of proteins. TMC nanoparticles have an excellent loading capacity for proteins, and a positive surface charge, suitable for attaching to nasal mucosa. In vivo experiments showed that TMC nanoparticles loaded with fluorescein isothiocyanatealbumin, when administered in the nasal cavity, were able to cross the mucosal layer, be taken up by rat nasal epithelia and NALT cells, and transported to submucosal layers. TMC nanoparticles are a potential new delivery system for protein transport through the nasal mucosa. ${ }^{72}$ Wang et al, ${ }^{73}$ who prepared estradiol-loaded chitosan nanoparticles and investigated the levels of estradiol in blood and cerebrospinal fluid in rats after intranasal administration, showed that estradiol levels in the cerebrospinal fluid after intranasal administration were significantly higher than after intravenous administration. The drug targeting index (DTI) of the nasal route was 3.2 and drug targeting percent (DTP\%) was $68.4 \% .^{73}$ The combination of bioadhesion and paracellular transport effects has led to chitosan to be considered for the delivery of estradiol via the nasal cavity. Huo et al, ${ }^{74}$ who used N-octyl-O-glycol chitosan (OGC) as the carrier of paclitaxel for intravenous administration, found that OGC for intravenous administration had good biocompatibility and no toxicity. Moreover, paclitaxel-loaded OGC micelles had low toxicity and a higher tolerated dose. In vivo studies of chitosan-fluorescent nanoparticles (CS-fl) prepared for ocular administration showed that the amounts of CS-fl in cornea and conjunctiva were significantly higher for CS-fl nanoparticles than for a control CS-fl solution, these amounts being fairly constant for up to 24 hours. $^{75}$

\section{Future prospects}

As a drug delivery system, chitosan nanoparticles have attracted increasing attention because of their good biocompatibility, degradability, and nontoxicity. Absorption and bioavailability of drug encapsulated into chitosan nanoparticles can be improved, so they can be used to deliver 
protein drugs, gene drugs, and other drugs and can protect them effectively from enzyme degradation in vivo. Chitosan nanoparticles are now being modified for sustained/ controlled release and targeting. As the active antitumor components of plant drugs are being constantly discovered and developed, developing targeted chitosan carriers for sustained/controlled release plant drugs is also an area of future development. While great progress has been achieved in the application of chitosan nanoparticles as drug carriers, some problems remain to be resolved urgently. For example, chitosan has poor solubility and unmodified chitosan nanoparticles can encapsulate only some hydrophilic drugs. Although chitosan can be modified easily to encapsulate hydrophobic drugs, further investigation is required on the biocompatibility of modified chitosan and its derivatives. In conclusion, chitosan and its derivatives as drug carriers have potential for a wider application.

\section{Acknowledgments}

This work was supported by grants from the Foundation of Zhejiang Science and Technology Department (2009C33005), National Natural Science Foundation of China (81001647), China Postdoctoral Science Foundation (20100471757), and National Natural Science Foundation of China (20906016).

\section{Disclosure}

The authors report no conflicts of interest. The authors are solely responsible for the content and writing of the article.

\section{References}

1. Shi XY, Fan XG. Advances in nanoparticle system for deliverying drugs across the biological barriers. J China Pharm Univ. 2002;33(3): 169-172.

2. Jin $\mathrm{MX}, \mathrm{Hu} \mathrm{QH}$. Characterization and application in bioadhesive drug delivery system of chitosan. Centr South Pharm. 2008;6(003): 324-327.

3. Dudhani AR, Kosaraju SL. Bioadhesive chitosan nanoparticles: preparation and characterization. Carbohydr Polym. 2010;81(2):243-251.

4. Kean T, Thanou M. Biodegradation, biodistribution and toxicity of chitosan. Adv Drug Deliv Rev. 2010;62(1):3-11.

5. Wedmore I, McManus J, Pusateri A, Holcomb J. A special report on the chitosan-based hemostatic dressing: experience in current combat operations. J Trauma. 2006;60(3):655-658.

6. Cao J, Zhou NJ. Progress in antitumor studies of chitosan. Chin J Biochem Pharm. 2005;26(2):127-127.

7. Maeda Y, Kimura Y. Antitumor effects of various low-molecular-weight chitosans are due to increased natural killer activity of intestinal intraepithelial lymphocytes in sarcoma 180-bearing mice. J Nutr. 2004; 134(4):945-950.

8. Torzsas T, Kendall C, Sugano M, Iwamoto Y, Rao A. The influence of high and low molecular weight chitosan on colonic cell proliferation and aberrant crypt foci development in CF1 mice. Food Chem Toxicol. 1996;34(1):73-77.
9. Zhou SH, Hong Y, Fang GJ. Preparation, characterization and anticancer effect of chitosan nanoparticles. J Clin Rehab Tiss Engin Res. 2007; 11(48):9688-9691.

10. Fang GJ, Hong Y, Jiang YY. Comparison of antitumor effects of chitosan nanoparticles from different sources in vitro. J Clin Rehab Tiss Engin Res. 2007;11(48):9696-9699.

11. Amidi M, Mastrobattista E, Jiskoot W, Hennink WE. Chitosan-based delivery systems for protein therapeutics and antigens. Adv Drug Deliv Rev. 2010;62(1):59-82.

12. Avadi MR, Sadeghi AMM, Mohammadpour N, et al. Preparation and characterization of insulin nanoparticles using chitosan and Arabic gum with ionic gelation method. Nanomed Nanotechnol Biol Med. 2010;6(1): 58-63.

13. Du W, Niu S, Xu Y, Xu Z, Fan C. Antibacterial activity of chitosan tripolyphosphate nanoparticles loaded with various metal ions. Carbohydr Polym. 2009;75(3):385-389.

14. Prabaharan M, Mano J. Chitosan-based particles as controlled drug delivery systems. Drug Deliv. 2004;12(1):41-57.

15. Ohya Y, Shiratani M, Kobayashi H, Ouchi T. Release behavior of 5-fluorouracil from chitosan-gel nanospheres immobilizing 5-fluorouracil coated with polysaccharides and their cell specific cytotoxicity. J Macromol Sci Part A. 1994;31(5):629-642.

16. Goldberg M, Langer R, Jia X. Nanostructured materials for applications in drug delivery and tissue engineering. J Biomater Sci Polym Ed. 2007; 18(3):241-268.

17. Bodnar M, Hartmann J, Borbely J. Preparation and characterization of chitosan-based nanoparticles. Biomacromolecules. 2005;6(5): 2521-2527.

18. Qu J, Liu G, Wang Y, Hong R. Preparation of $\mathrm{Fe}_{3} \mathrm{O}_{4}$-chitosan nanoparticles used for hyperthermia. Adv Powder Technol. 2010;21(4):461-467.

19. Berthold A, Cremer K, Kreuter J. Preparation and characterization of chitosan microspheres as drug carrier for prednisolone sodium phosphate as model for anti-inflammatory drugs. J Control Release. 1996; 39(1):17-25.

20. Tian X, Groves M. Formulation and biological activity of antineoplastic proteoglycans derived from Mycobacterium vaccae in chitosan nanoparticles. J Pharm Pharmacol. 1999;51(2):151-157.

21. El-Shabouri M. Positively charged nanoparticles for improving the oral bioavailability of cyclosporin-A. Int J Pharm. 2002;249(1-2):101-108.

22. Chauvierre C, Labarre D, Couvreur P, Vauthier C. Radical emulsion polymerization of alkylcyanoacrylates initiated by the redox system dextran-cerium (IV) under acidic aqueous conditions. Macromolecules. 2003;36(16):6018-6027.

23. Chauvierre C, Labarre D, Couvreur P, Vauthier C. Plug-in spectrometry with optical fibers as a novel analytical tool for nanoparticles technology: application to the investigation of the emulsion polymerization of the alkylcyanoacrylate. J Nanopart Res. 2003;5(3):365-371.

24. Chauvierre C, Labarre D, Couvreur P, Vauthier C. Novel polysaccharidedecorated poly (isobutyl cyanoacrylate) nanoparticles. Pharm Res. 2003;20(11):1786-1793.

25. Bravo-Osuna I, Vauthier C, Farabollini A, Palmieri GF, Ponchel G. Mucoadhesion mechanism of chitosan and thiolated chitosan-poly(isobutyl cyanoacrylate) core-shell nanoparticles. Biomaterials. 2007;28(13): 2233-2243.

26. de Moura MR, Aouada FA, Mattoso LHC. Preparation of chitosan nanoparticles using methacrylic acid. J Colloid Interface Sci. 2008; 321(2):477-483.

27. Kim JH, Kim YS, Park K, et al. Antitumor efficacy of cisplatin-loaded glycol chitosan nanoparticles in tumor-bearing mice. J Control Release. 2008;127(1):41-49.

28. Huo MR, Zhou JP, Zhang Y. Synthesis and characterization of novel amphiphilic chitosan derivatives and its solubilizing abilities for waterinsoluble drugs. Chem J Chinese U. 2007;28(10):1995-1999.

29. Cheng MH, Huang YX, Zhou HJ, Liu Z, Li JF. Rapid preparation and characterization of chitosan nanoparticles for oligonucleotide. Curr Appl Phys. 2010;10(3):797-800. 
30. Kim D, Jeong Y, Choi C, et al. Retinol-encapsulated low molecular watersoluble chitosan nanoparticles. Int J Pharm. 2006;319(1-2):130-138.

31. Grenha A, Seijo B, Serra C, Remu án-López C. Chitosan nanoparticleloaded mannitol microspheres: structure and surface characterization. Biomacromolecules. 2007;8(7):2072-2079.

32. Huang HY, Shieh YT, Shih CM, Twu YK. Magnetic chitosan/iron (II, III) oxide nanoparticles prepared by spray-drying. Carbohydr Polym. 2010;81(4):906-910.

33. Singh R, Lillard J Jr. Nanoparticle-based targeted drug delivery. Exp Mol Pathol. 2009;86(3):215-223.

34. Yuan Q, Shah J, Hein S, Misra RDK. Controlled and extended drug release behavior of chitosan-based nanoparticle carrier. Acta Biomater. 2010;6(3):1140-1148.

35. Park JH, Saravanakumar G, Kim K, Kwon IC. Targeted delivery of low molecular drugs using chitosan and its derivatives. Adv Drug Deliv Rev. 2010;62(1):28-41.

36. Park K, Kim J, Nam Y, et al. Effect of polymer molecular weight on the tumor targeting characteristics of self-assembled glycol chitosan nanoparticles. J Control Release. 2007;122(3):305-314.

37. Xu XY, Zhou JP, Li L. Preparation of doxorubicin-loaded chitosan polymeric micelle and study on its tissue biodistribution in mice. Acta Pharm Sin. 2008;43(7):743-748.

38. Jones R, Cheung C, Black F, et al. Poly (2-alkylacrylic acid) polymers deliver molecules to the cytosol by $\mathrm{pH}$-sensitive disruption of endosomal vesicles. Biochem J. 2003;372(Pt 1):65-75.

39. Kiang T, Bright C, Cheung C, Stayton P, Hoffman A, Leong K. Formulation of chitosan-DNA nanoparticles with poly (propyl acrylic acid) enhances gene expression. J Biomater Sci, Polym Ed. 2004;15(11): 1405-1421.

40. Fan L, Wu H, Zhang $\mathrm{H}$, et al. Novel super $\mathrm{pH}$-sensitive nanoparticles responsive to tumor extracellular pH. Carbohydr Polym. 2008;73(3): 390-400.

41. Pan A, Wu B, Wu J. Chitosan nanoparticles crosslinked by glycidoxypropyltrimethoxysilane for $\mathrm{pH}$ triggered release of protein. Chin Chem Lett. 2009;20(1):79-83.

42. Chung J, Yokoyama M, Aoyagi T, SakuraiY, Okano T. Effect of molecular architecture of hydrophobically modified poly ( $\mathrm{N}$-isopropylacrylamide) on the formation of thermoresponsive core-shell micellar drug carriers. J Control Release. 1998;53(1-3):119-130.

43. Chung J, Yokoyama M, Yamato M, Aoyagi T, Sakurai Y, Okano T. Thermo-responsive drug delivery from polymeric micelles constructed using block copolymers of poly (N-isopropylacrylamide) and poly (butylmethacrylate). J Control Release. 1999;62(1-2):115-127.

44. Rejinold NS, Chennazhi KP, Nair SV, Tamura H, Jayakumar R. Biodegradable and thermo-sensitive chitosan-g-poly(N-vinylcaprolactam) nanoparticles as a 5-fluorouracil carrier. Carbohydr Polym. 2011;83(2): 776-786.

45. Yao Q. Study on the two-ligand modified chitosan nanoparticles actively targeting to malignant liver cells. Doctoral Paper of Sichuan University 2006.

46. Huang Y, Lin AH, Zhang X. Targeting binding of chitosan nanoparticles with glycyrrhizin surface modification to hepatic parenchymal cells in vitro. Tradit Chin Drug Res Pharmacol. 2008;19(6):495-498.

47. Min K, Park K, Kim Y, et al. Hydrophobically modified glycol chitosan nanoparticles-encapsulated camptothecin enhance the drug stability and tumor targeting in cancer therapy. J Control Release. 2008;127(3):208-218.

48. Mei ZN, Yang XL, Xu HB. Biodegradable polymer long-circulating nanoparticle. Chin J Hosp Pharm. 2002;22(7):433-435.

49. Redhead H, Davis S, Illum L. Drug delivery in poly (lactide-coglycolide) nanoparticles surface modified with poloxamer 407 and poloxamine 908: in vitro characterisation and in vivo evaluation. J Control Release. 2001;70(3):353-363.

50. Nam HY, Kwon SM, Chung H, et al. Cellular uptake mechanism and intracellular fate of hydrophobically modified glycol chitosan nanoparticles. J Control Release. 2009;135(3):259-267.
51. Yang YM, Hu W, Wang XD, Gu XS. The controlling biodegradation of chitosan fibers by $\mathrm{N}$-acetylation in vitro and in vivo. J Mater Sci: Mater Med. 2007;18(11):2117-2121.

52. Xu J, McCarthy S, Gross R, Kaplan D. Chitosan film acylation and effects on biodegradability. Macromolecules. 1996;29(10):3436-3440.

53. Jayakumar R, Chennazhi KP, Muzzarelli RAA, Tamura H, Nair SV, Selvamurugan N. Chitosan conjugated DNA nanoparticles in gene therapy. Carbohydr Polym. 2010;79(1):1-8.

54. Katas H, Alpar H. Development and characterization of chitosan nanoparticles for siRNA delivery. $J$ Control Release. 2006;115(2): 216-225.

55. Liu XD. Chitosan-siRNA complex nanoparticles for gene silencing. J Biomed Engin. 2010;27(1):97-101.

56. Mao S, Sun W, Kissel T. Chitosan-based formulations for delivery of DNA and siRNA. Adv Drug Deliv Rev. 2010;62(1):12-27.

57. Lavertu M, Methot S, Trankhanh N, Buschmann M. High efficiency gene transfer using chitosan/DNA nanoparticles with specific combinations of molecular weight and degree of deacetylation. Biomaterials. 2006;27(27):4815-4824.

58. Mansouri S, Cuie Y, Winnik F, et al. Characterization of folatechitosan-DNA nanoparticles for gene therapy. Biomaterials. 2006; 27(9):2060-2065.

59. Gan Q, Wang T. Chitosan nanoparticle as protein delivery carriersystematic examination of fabrication conditions for efficient loading and release. Colloids Surf B. 2007;59(1):24-34.

60. Zhang N, Li J, Jiang W, et al. Effective protection and controlled release of insulin by cationic $\beta$-cyclodextrin polymers from alginate/chitosan nanoparticles. Int J Pharm. 2010;393(1-2):213-219.

61. Kim JH, Kim YS, Park K, et al. Self-assembled glycol chitosan nanoparticles for the sustained and prolonged delivery of antiangiogenic small peptide drugs in cancer therapy. Biomaterials. 2008;29(12): 1920-1930.

62. Jeong YI, Jin SG, Kim IY, et al. Doxorubicin-incorporated nanoparticles composed of poly(ethylene glycol)-grafted carboxymethyl chitosan and antitumor activity against glioma cells in vitro. Colloids Surf B. 2010;79(1):149-155.

63. Li F, Li J, Wen X, et al. Anti-tumor activity of paclitaxel-loaded chitosan nanoparticles: An in vitro study. Mater Sci Eng, C. 2009;29(8): 2392-2397.

64. Trickler WJ, Nagvekar AA, Dash AK. A novel nanoparticle formulation for sustained paclitaxel delivery. AAPS Pharm Sci Tech. 2008;9(2): 486-493.

65. Kim JH, Kim YS, Kim S, et al. Hydrophobically modified glycol chitosan nanoparticles as carriers for paclitaxel. $J$ Control Release. 2006;111(1-2):228-234.

66. Hao PP, Deng SH. Preparation and detection of acyclovir loaded chitosan nanoparticles. Chin Med Herald. 2008;5(1):28-29.

67. Li FB, Luan LB. Preparation and in vitro release of tranilast-loaded chitosan nanoparticles. Pharm Clin Res. 2008;16(4):282-284.

68. Samstein R, Perica K, Balderrama F, Look M, Fahmy T. The use of deoxycholic acid to enhance the oral bioavailability of biodegradable nanoparticles. Biomaterials. 2008;29(6):703-708.

69. Yin L, Ding J, He C, Cui L, Tang C, Yin C. Drug permeability and mucoadhesion properties of thiolated trimethyl chitosan nanoparticles in oral insulin delivery. Biomaterials. 2009;30(29):5691-5700.

70. Dube A, Nicolazzo JA, Larson I. Chitosan nanoparticles enhance the intestinal absorption of the green tea catechins $(+)$-catechin and (-)-epigallocatechin gallate. Eur J Pharm Sci. 2010;41(2): 219-225.

71. Makhlof A, Tozuka Y, Takeuchi H. Design and evaluation of novel pHsensitive chitosan nanoparticles for oral insulin delivery. Eur J Pharm Sci. 2010; doi:10.1016/j.ejps. 2010.12.007. In press.

72. Amidi M, Romeijn S, Borchard G, Junginger H, Hennink W, Jiskoot W. Preparation and characterization of protein-loaded N-trimethyl chitosan nanoparticles as nasal delivery system. J Control Release. 2006; 111(1-2):107-116. 
73. Wang X, Chi N, Tang X. Preparation of estradiol chitosan nanoparticles for improving nasal absorption and brain targeting. Eur J Pharm Biopharm. 2008;70(3):735-740.

74. Huo M, Zhang Y, Zhou J, et al. Synthesis and characterization of lowtoxic amphiphilic chitosan derivatives and their application as micelle carrier for antitumor drug. Int J Pharm. 2010;394(1-2):162-173.
75. De Campos A, Diebold Y, Carvalho E, Sanchez A, Jose Alonso M. Chitosan nanoparticles as new ocular drug delivery systems: in vitro stability, in vivo fate, and cellular toxicity. Pharm Res. 2004;21(5): 803-810.

\section{Publish your work in this journal}

The International Journal of Nanomedicine is an international, peerreviewed journal focusing on the application of nanotechnology in diagnostics, therapeutics, and drug delivery systems throughout the biomedical field. This journal is indexed on PubMed Central, MedLine, CAS, SciSearch $\AA$, Current Contents ${ }^{\circledR} /$ Clinical Medicine,
Journal Citation Reports/Science Edition, EMBase, Scopus and the Elsevier Bibliographic databases. The manuscript management system is completely online and includes a very quick and fair peer-review system, which is all easy to use. Visit http://www.dovepress.com/ testimonials.php to read real quotes from published authors.

Submit your manuscript here: http://www.dovepress.com/international-journal-of-nanomedicine-journal 